\title{
Cooperative emergency braking warning system in vehicular networks
}

\author{
Ming-Fong Tsai ${ }^{1}$, Yung-Cheng Chao ${ }^{2}$, Lien-Wu Chen ${ }^{1}$, Naveen Chilamkurti ${ }^{3}$ and Seungmin Rho $^{4^{*}}$
}

\begin{abstract}
Safety applications in vehicular networks have been popular research topics in recent years, such as forward collision warning, emergency braking warning and intersection collision warning systems. The basic safety message broadcast from each car transmits the position, car speed and car heading information. Neighbouring cars receiving this information can decide if there is any danger within the next second. However, the safety application message has positioning accuracy and time-critical problems. Accurate positioning of the car provides a more effective warning to the driver. Moreover, the reliable and efficient delivery of safety information needs to be improved when the penetration rate increases. Hence, this paper proposes a cooperative emergency braking warning system to solve the above problems. This is a system that integrates a camera sensor to construct the state of neighbourhood cars in order to solve the first problem. It then proposes to reduce the repeat and derivable information between the broadcast messages in order to solve the second problem. The proposed system has been implemented to provide a safer driving environment.
\end{abstract}

Keywords: Safety applications; Cooperative system; Vehicular networks

\section{Introduction}

More and more research works and products have been proposed in recent years for vehicle-to-vehicle or vehicleto-roadside communication for reducing accidents [1-5]. Based on previous research, protocol standards and field trial works, the US Department of Transportation has published information announcing a decision to move forward with vehicle-to-vehicle communication technology for light vehicles in 2014 [6]. The typical safety application in the vehicular networks is the emergency electronic brake light (EEBL) [7], as shown in Figure 1. The second car may stop without collision when the first car brakes hard, based on driver vision, but the third car cannot stop fast enough and collides with the second car. If the cars had vehicle-tovehicle communication devices, such as ITRI WAVE/ DSRC Communications Units (IWCUs) [8], each car could broadcast basic safety message (BSM) [9] information to warn cars in the neighbourhood. Hence, the third car would receive the broadcast BSM information from the

\footnotetext{
* Correspondence: korea.smrho@gmail.com

${ }^{4}$ Department of Multimedia, Sungkyul University, Anyang, Korea

Full list of author information is available at the end of the article
}

first car; and based on this information, the third car would decide whether to alert the driver.

There are two problems that need to be solved for safety applications in the vehicular networks. The first is the positioning accuracy issue, and the second is the time-critical issue. For vehicular networks, the safety applications of the broadcast BSM information frequency have been described in the WAVE/DSRC provided environment [10]. The car identity and car position in the warning information are based on the Global Positioning System (GPS). There are many enhanced technologies for GPS, such as assisted GPS, differential GPS and the wide-area augmentation system. However, these enhanced technologies cannot work at lane level in a city [11]. Hence, the GPS cannot identify the position of the car very well in recent research. The broadcast frequency of many safety applications requires a minimum of $10 \mathrm{~Hz}$ (maximum latency is $100 \mathrm{~ms}$ ), such as for emergency brake lights, traffic signal violation, forward collision, left turn assist and lanechange warning. More and more channel collisions have happened, and larger latency problems will happen when the WAVE/DSRC device penetration rate increases. Controlling the transmission power [12] and transmission frequency [13] to avoid affecting neighbouring cars are two

\section{Springer}




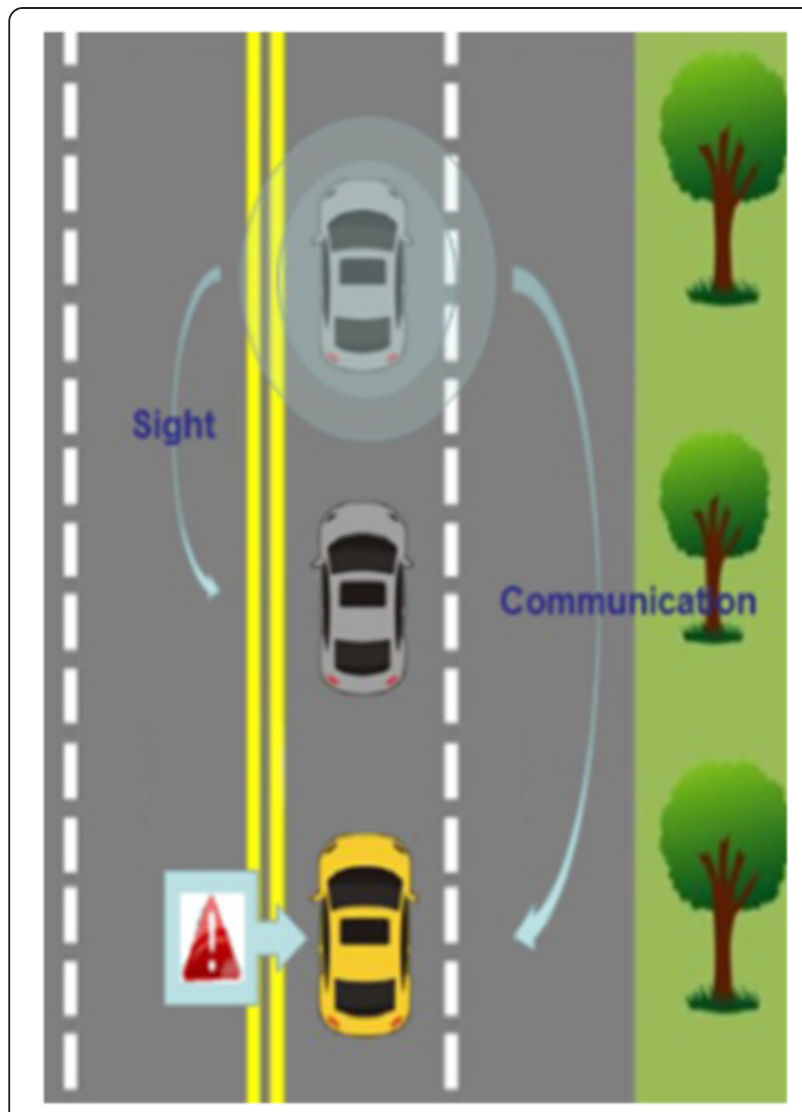

Figure 1 Emergency electronic brake light system.

angle methods to overcome the time-critical issue. However, these two angle methods need to obtain more information to decide the optimal rate or frequency control, such as feedback information from neighbouring cars, or to monitor the broadcast condition immediately.

This paper proposes a cooperative emergency braking warning system which integrates a camera sensor to construct the state of neighbouring cars. The proposed system uses the relative position instead of the GPS position in order to solve the positioning accuracy problem. This method will not affect the GPS operation but enhances the above research works. The proposed system uses previous work [14] which reduces the repeated and derivable information between the broadcast messages in order to solve the time-critical problem. This method will not change the transmission power and frequency but is compatible with the above research works. This paper is structured as follows. Section 2 presents a brief overview of the background and related works. Section 3 presents the proposed cooperative emergency braking warning system. Implementation performance evaluations are presented in Section 4. Conclusions and future work are given in Section 5.

\section{Background and related works \\ 2.1 Background}

With vehicle-to-vehicle communication, drivers can give an earlier braking warning signal when the vehicle in front of the vehicle ahead emergency brakes, especially when the front view is obstructed by another vehicle. However, there are still some challenges that need to be overcome for vehicle-to-vehicle communication.

\subsubsection{Positioning accuracy}

Unlike other wireless communication, vehicle-to-vehicle communication performs well especially in a low-latency and highly mobility time-varying environment. Traditionally, most related work assumes that both the emergency braking signal and the GPS position are broadcast, and the received vehicle will check the source of the emergency braking signal according to the GPS position. If the heading matches and the GPS position 'seems' on the same lane, a warning signal is received. However, this is not accurate enough to detect the lane by GPS alone. Although position technologies like assisted GPS, differential GPS and the wide-area augmentation system can improve positioning accuracy, in an environment like a downtown area, there is usually severe shadowing due to buildings, trees, vehicles and tunnels. Consequently, the position performance may not be effective at lane level. As shown in Figure 2, when car E emergency brakes, this warning signal will be broadcast and received by vehicles behind in the same lane. Since the broadcasting range of DSRC can be up to 300 metres, the surrounding vehicles, including in the opposite lane, will also receive the warning signal at the same time. This will cause some trouble and unnecessary interference to vehicles $A, B, C, I, J$ and $K$ when the range of GPS error is greater.

\subsubsection{Transmission latency}

The IEEE 1609 (WAVE/DSRC) standard allocates seven channels (10 MHz each) in a 5.9-GHz band for communication between vehicle-to-vehicle or vehicle-to-roadside devices [15]. The device on the car is called the On-Board Unit (OBU), which needs to be shockproof, and the device on the roadside is called the Road-Side Unit (RSU), which needs to be waterproof. OBU to OBU or OBU to RSU communication is called dedicated short-range communication (DSRC). The control channel $(\mathrm{CCH})$ is used to transmit the control or safety message in the vehicular network, such as BSM information. The BSM information is used in safety applications to exchange data regarding the car state. The BSM information is broadcast frequently to neighbouring cars with a variety of information required for safety applications. Part I of the BSM information is included in every broadcast BSM packet, while part II of the BSM information is optional, as shown in 


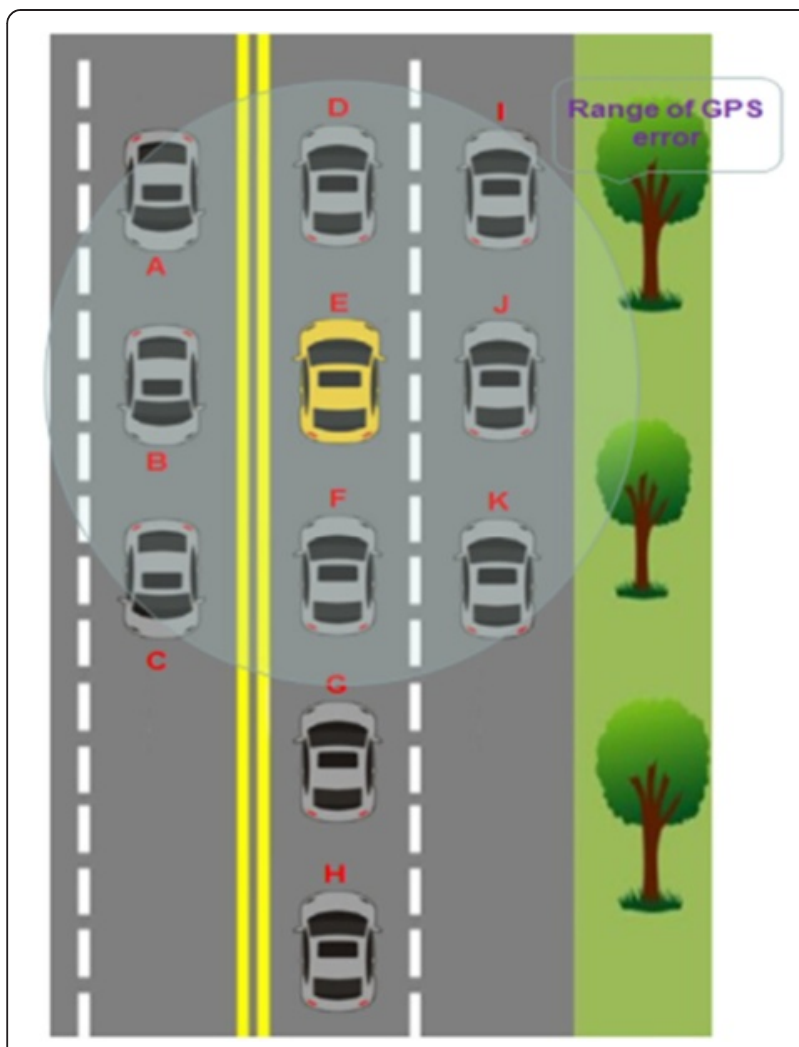

Figure $\mathbf{2}$ Challenges of position error in emergency braking warning system (cars $[\mathrm{A}-\mathrm{K}]$ ).

Figure 3. A broadcast storm problem arises when all the vehicles on the road are broadcasting their state and position information at $10 \mathrm{~Hz}$. Moreover, if a vehicle emergency brakes, vehicles in the same lane behind have to be informed with the warning signal. Since the coverage of DSRC is limited to about 300 metres, the system will forward a rebroadcast to inform vehicles in the same lane that are out of communication coverage.

\subsection{Related works}

The proposed cooperative emergency braking warning system should solve two issues in the vehicular networks. The first part of the related works is the positioning accuracy issue, and the second is the transmission latency issue.

\subsubsection{Positioning accuracy}

Many enhanced positioning accuracy technologies have been proposed. The differential GPS and wide-area augmentation systems have been used to enhance the accuracy of GPS in recent years. The differential GPS technology uses a network of fixed, ground-based reference stations broadcasting the difference value between the positions indicated by the satellite systems and the

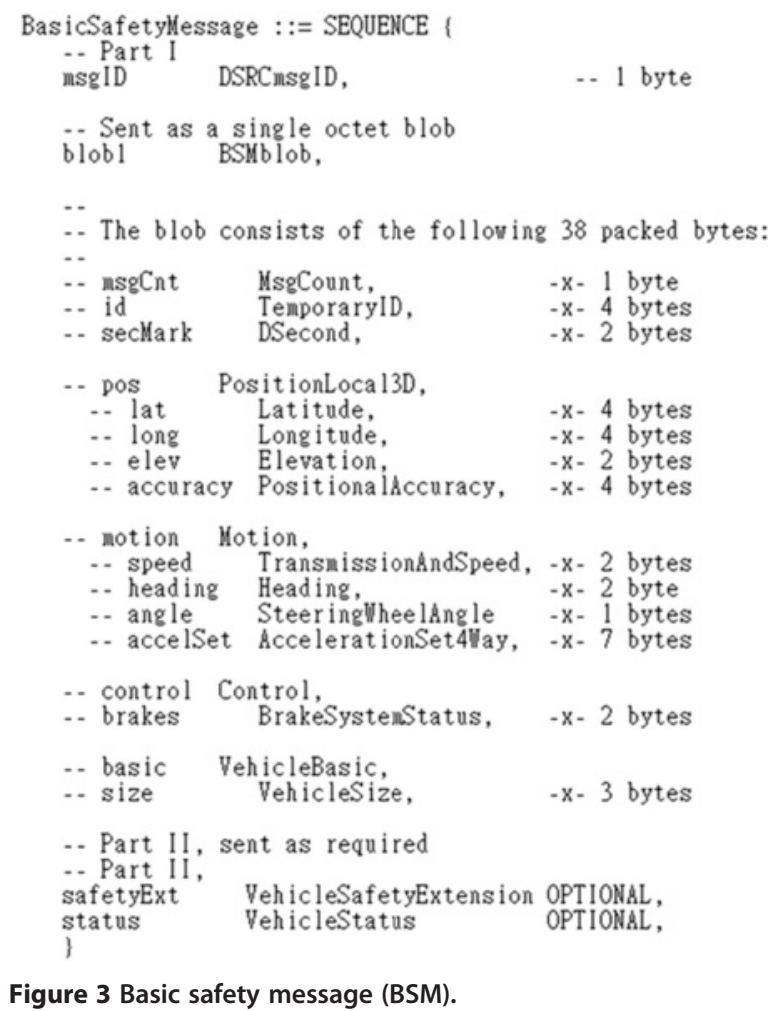

Figure 3 Basic safety message (BSM).

known fixed positions [16]. The wide-area augmentation systems technology uses a network of groundbased reference stations to measure small variations in the GPS signals. These satellites broadcast correction information back to the Earth, where wide-area augmentation system receivers use the correction value while computing their positions to improve accuracy [17]. However, these differential GPS and wide-area augmentation system technologies are occasionally incorrect because of obstructions and multipath effects caused by buildings and vegetation. This paper's proposed system uses the relative position instead of the GPS position in order to enhance the positioning accuracy. The proposed system will not affect GPS operation but enhance the above research works.

\subsubsection{Transmission latency}

Many research works in recent years have sought to overcome transmission latency. These works proposed broadcast transmission frequency adjustment methods to overcome this issue $[13,18,19]$. Moreover, many other research works have proposed broadcast transmission power adjustment methods to overcome the problem $[12,20,21]$. However, not only transmission frequency adjustment methods but also transmission power adjustment methods are needed to obtain more information, 
to decide the optimal rate or frequency control, such as feedback information from neighbouring cars, or to monitor the broadcast condition immediately. This paper proposes a system that reduces the repeated and derivable information between the broadcast messages, thus solving the time-critical issue. The proposed system will not change the transmission power and frequency but is compatible with the above research works.

\section{Proposed method}

\subsection{System model of cooperative emergency braking} warning system

The main objective of the proposed cooperative emergency braking warning system is to provide drivers with a braking warning signal without the obscurity due to weather factors. The proposed braking warning system combines dedicated short-range communication (DSRC) and image recognition technology to minimize the positioning error from vehicle-to-vehicle communication. With vehicle-to-vehicle communication, drivers can achieve higher braking warning accuracy when the vehicle ahead of the vehicle in front emergency brakes, especially in bad weather or when the front view is obstructed by another vehicle. Moreover, the proposed system changes the message transmission coverage based on the distance and direction from the emergency braking vehicle and different weather factors. In this way, drivers can eliminate unnecessary warnings and achieve a more accurate braking warning quality.

\subsubsection{Vehicle collision warning}

Take three cars A, B and C in the same lane and the same driving direction; for example, if car A emergency brakes, the braking distance and time can be described as shown in Equation 1. In Figure 4, $\mathrm{t}_{\mathrm{w}}$ is the warning time of car C's driver, which is also the time when car B's braking light is turned on. $t_{b}$ represents the initial braking timing of car C. $t_{\text {stop }}$ is the stop time of car C. The reaction time is $T_{r}=t_{b}-t_{w}$. From research, people's reaction time differs in the range of 0.7 to $1.5 \mathrm{~s}$.

$$
d_{w}=v_{f} T_{r}+\frac{v_{f}^{2}}{2 a_{f}}-\frac{v_{p}^{2}}{2 a_{p}}+d_{\text {stop }}
$$

where $d_{w}$ is the distance between car B and car C when car B's braking light is turned on. $\mathrm{v}_{\mathrm{f}}$ is the velocity of car $C$, and $v_{p}$ is the velocity of car $B$. $a_{f}$ is the acceleration of car C, and $a_{p}$ is the acceleration of car B. $d_{\text {stop }}$ is the distance between car B and car $C$ at the final state. $T_{r}$ is the reaction time from seeing the braking light of car $\mathrm{B}$ to stopping. We can derive from Equation 1 that the collision occurs if $d_{\text {stop }}$ is less than or equal to zero. To avoid collision, we can apply the following methods.
1. Enlarge $d_{w}$ : the greater the distance from the car ahead, the more reaction time drivers have, so there is less chance that a collision occurs. Normally, the safety distance recommended is as Equation 2. However, this factor is not our concern in this paper as it is mainly controlled by drivers.

$d_{\text {safe }}(m)=\frac{v(\mathrm{~km} / \mathrm{h})}{2}$

2. Shorten the braking time: there is a large amount of vehicle research in this area, subject to different weather, roads, tyres and other effects, and it is already a mature technology.

3. Shorten the human reaction time: since more than $90 \%$ of car accidents are caused by the human factor, if drivers can receive a warning signal earlier by communication technology, they will have more time to deal with the emergency. According to research, if the warning signal pops up $0.5 \mathrm{~s}$ earlier, about $60 \%$ of car accidents can be avoided and the statistics improve further if it comes $1.5 \mathrm{~s}$ earlier, when more than $90 \%$ of car accidents can be avoided. This is also the paper's main concern.

The main contribution of the proposed cooperative emergency braking warning system delivers drivers a braking warning signal earlier by DSRC communication. Via vehicle-to-vehicle communication, the emergency braking warning signal will be broadcast and the transmission time is much shorter than the human reaction time. Therefore, drivers behind can receive an immediate emergency braking warning while the front vehicle emergency brakes. Since the transmission time is much shorter than the human reaction time, the driver in car $\mathrm{C}$ can be aware of the emergency braking of car A before the driver in car B brakes and its braking light comes on, as shown in Figure 4. Consequently, the early warning mechanism is the concept of the proposed cooperative emergency braking warning system. Now that active safety applications have become mature, cooperative communication technology to achieve improved safety will be the future trend.

\subsubsection{Vehicle list construction procedure}

This paper proposes a vehicle list construction mechanism taking message redundancy, transmission latency and reliability problem into consideration at the same time. Combined with image recognition technology, the system monitors the front view and applies the licence plate comparison technology to detect the front vehicles. The proposed system will utilize the vehicle speed, vehicle type and vehicle colour to increase the accuracy of the licence plate comparison so as to improve 


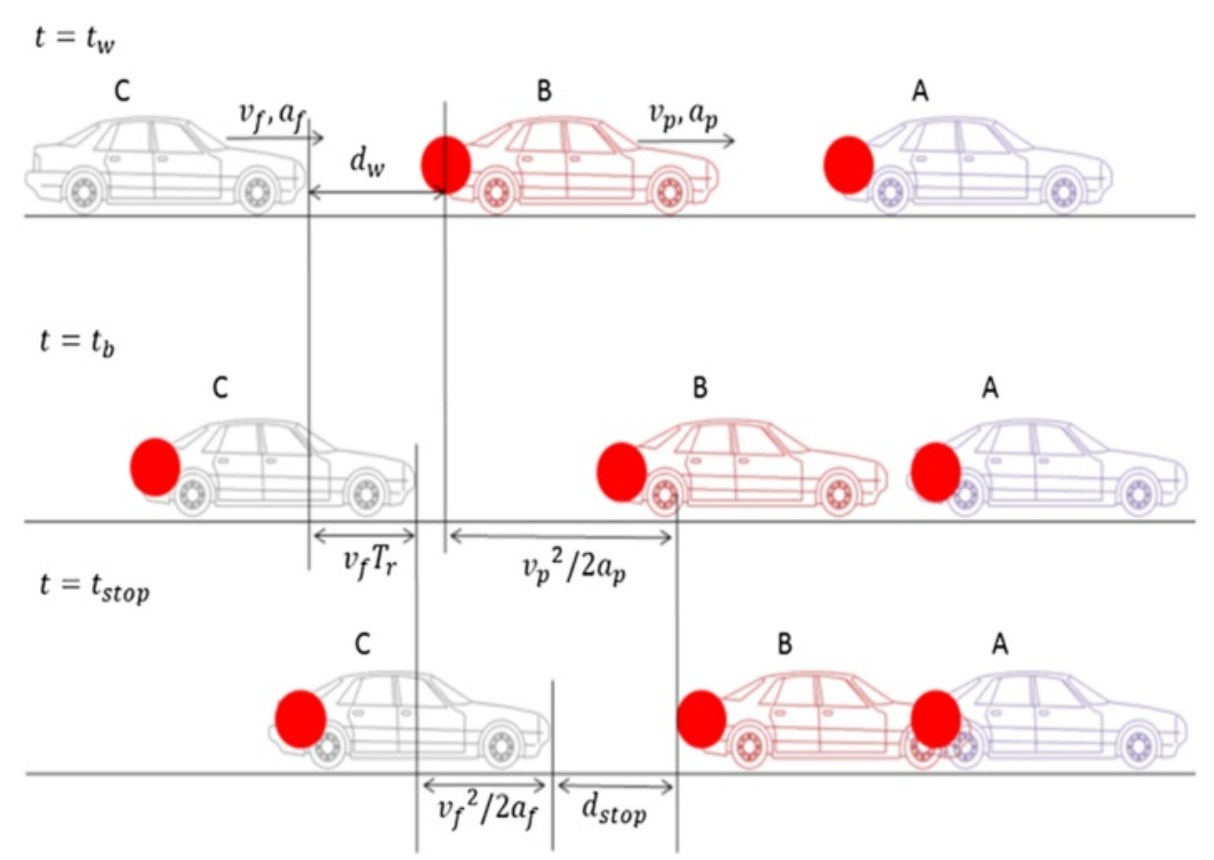

Figure 4 Braking kinematics analysis (cars $[A-C])$.

the position accuracy. The position accuracy of the proposed cooperative emergency braking warning system can be up to lane level. Once the front vehicle emergency brakes, vehicles behind in the same lane will receive the image and voice warning, but vehicles in different lanes will not receive the warning signal so as to avoid redundant interference with those drivers.

The cooperative emergency braking warning system architecture is shown in Figure 5. The proposed system includes an image detection and recognition module, safety message broadcasting module, vehicle list construction module and the user interface module. The image detection and recognition module makes use of the vehicle speed, vehicle type and vehicle colour to increase the detection accuracy of the licence plate in front of the driver's vehicle. The safety message and vehicle list are broadcast by DSRC communication. The proposed system uses the IWCU device. All the software programs developed have to be connected on the IWCU device. The IWCU device is a standard product completely compatible with IEEE 802.11p, IEEE1609 and SAE J2735 standard protocols. As shown in Figure 5, the software programs are integrated on the upper layer, while the lower layer is connected with the camera which serves as the input of image recognition. The accelerometer (G-sensor) or On-Board Diagnostics II (OBD-II) acts as the emergency braking detector; the GPS will support the rough range of the car's position. Moreover, the National Marine Electronics Association (NMEA) is the GPS standard protocol. Hence, each car can understand the car position information. In order to inform drivers immediately, the user interface is developed on the android system and connected with the IWCU by Wi-Fi. The user interface (UI) is responsible for the immediate warning condition display. As shown in Figure 6, the vehicle in our system is also equipped

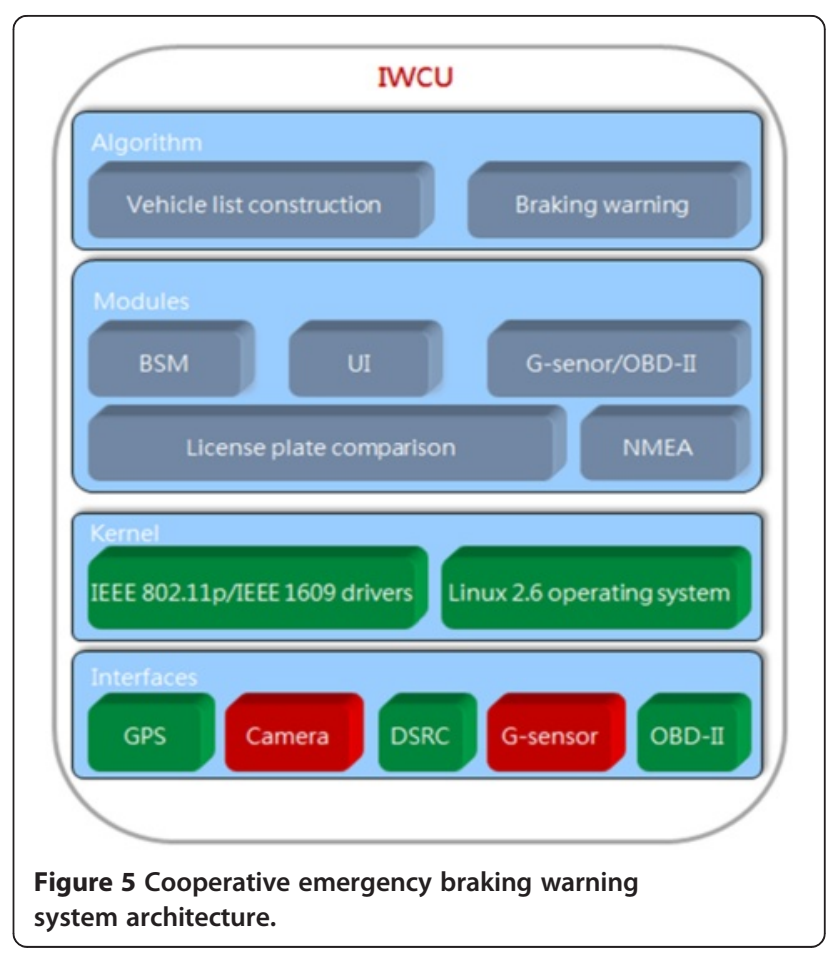




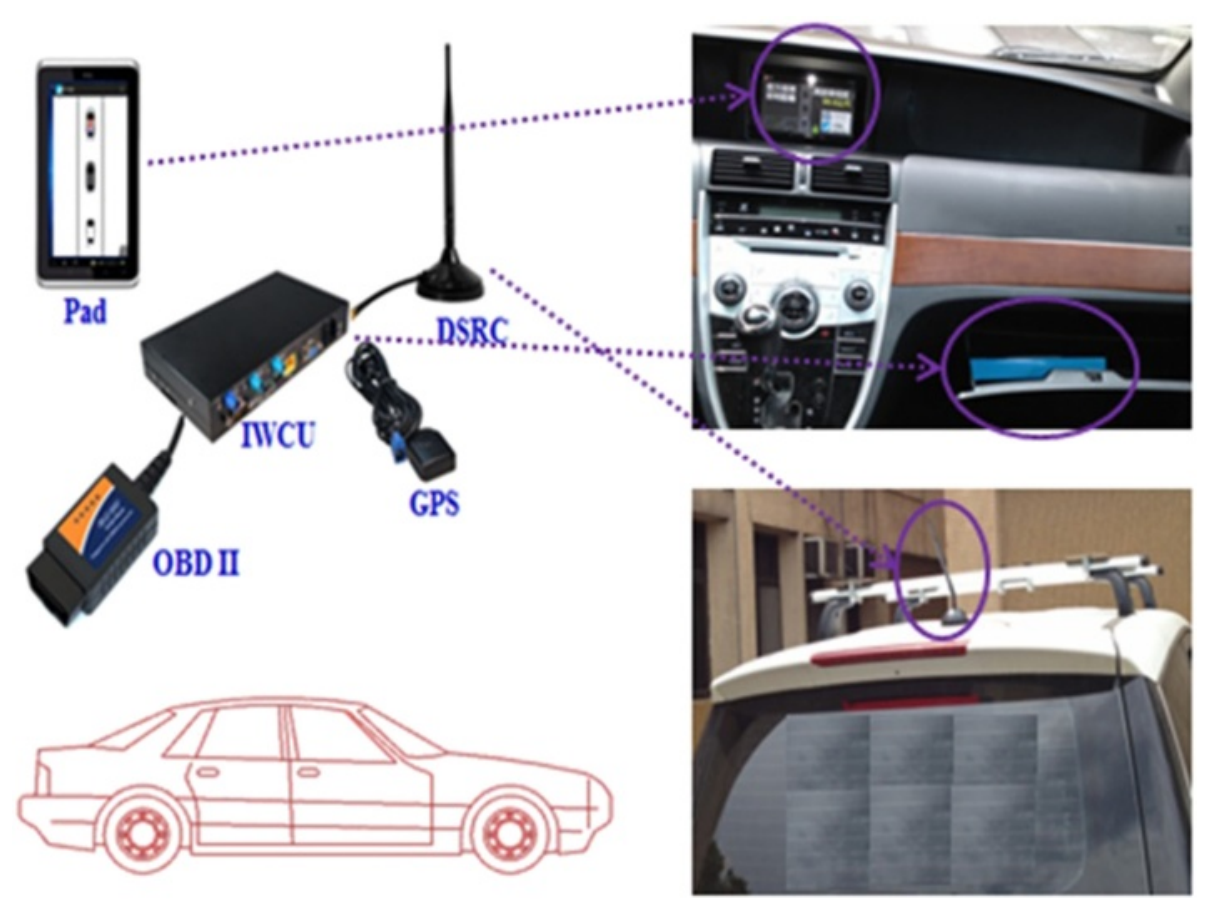

Figure 6 Cooperative emergency braking warning system equipment.

with an OBD-II or G-sensor to detect whether the vehicle is emergency braking or not.

The IWCU device on the vehicle broadcasts the BSM information, including the licence plate, vehicle colour, vehicle type, current GPS position and emergency events, periodically to other vehicles nearby. As they receive the BSM information, the licence plate comparison system will make use of the information collected and integrate the results from the camera sensor to determine the licence plates of the vehicles in front. The vehicle list construction module on the IWCU device will then put the licence plate information of the front vehicles into the BSM information. Therefore, the system will construct the list of vehicles in the same lane and in adjacent lanes. The vehicle list information can be constructed as shown in Figure 7. Each vehicle needs to broadcast the licence plate information, and a function of licence plate comparison is included. Licence plate recognition would therefore be successful even when there were character errors. There is more detailed information about the image recognition in our previous work [22]. The example of vehicle list construction is shown in Figure 8. The vehicle list construction module on the IWCU device will then input the licence plate information of the front vehicles into the BSM information. The second car uses the image information to identify the first car and input this information into the BSM information. Hence, the third car can obtain the information of the first and second cars having received the broadcast information from the second car. Moreover, the third car can use the image information to identify the second car and based on second to construct the first, second and third vehicle list. Furthermore, the warning information can be shown on the user interface (UI) for drivers, as shown in Figure 9, so that the drivers can be alerted straight away if any emergency event happens. The cooperative emergency braking warning system increases the position accuracy by integrating the image recognition technology. The accurate position of the vehicles on the list represents the real corresponding position of the vehicles around the driver at lane level. If a vehicle on the list emergency brakes, the driver can be alerted immediately by means of a voice signal and be informed what to do according to the warning condition and the relative position of the vehicle to the driver. The cooperative emergency braking warning system also works in cases when the view is obstructed or in bad weather.

The functional architecture of the proposed system is shown in Figure 10, and the algorithm comprises six steps:

1. The IWCU device on the vehicle periodically broadcasts the BSM information including the licence plate, vehicle colour, vehicle type, current GPS position, vehicle list and emergency events.

2. The licence plate comparison system and camera continuously monitor the front licence plates of the 

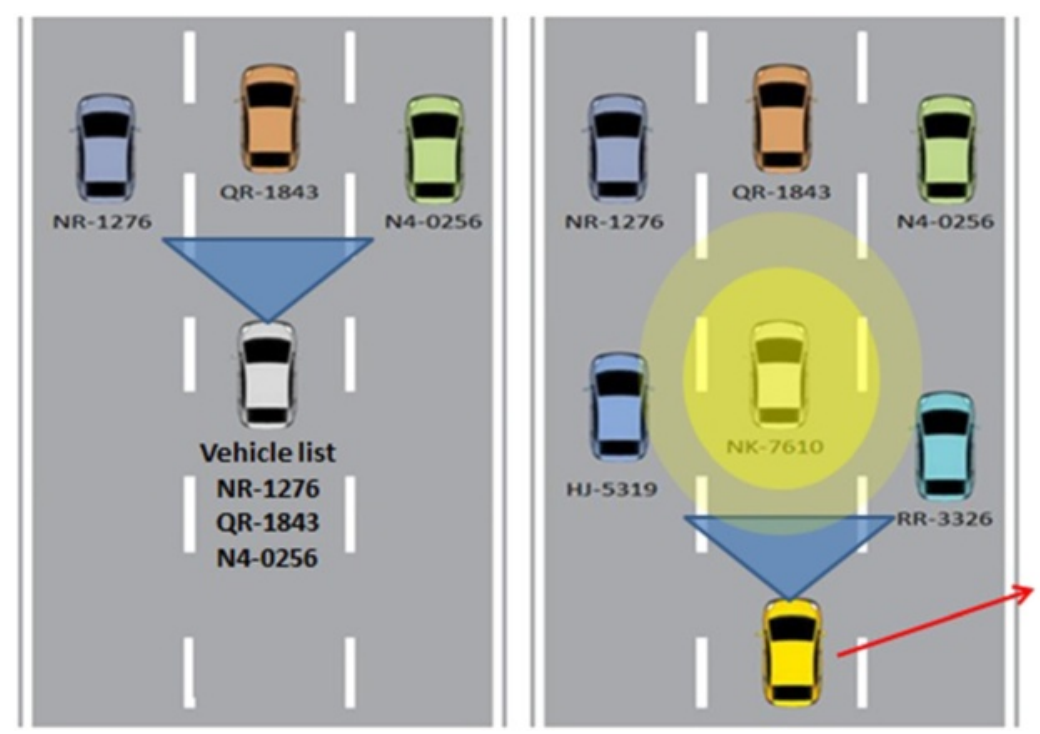

Vehicle list

NR-1276

QR-1843

N4-0256

HJ-5319

*NK-7610

RR-3326

Figure 7 Vehicle list construction result.

vehicles ahead. The detected result is used by the vehicle list construction system to update the vehicle list.

3. If there is an emergency braking warning signal, the vehicle list construction system will check whether the signal source is on the list or not.

4. The signal source is checked to determine the emergency level according to the corresponding position from the drivers to the source.
5. After the emergency level is determined, the system will show the emergency braking warning in terms of voice and image on the UI.

6. Return to step 1.

The cooperative emergency braking warning system combines image recognition technology with DSRC communication and applies the licence plate comparison technology to detect the vehicles in front so as to achieve high

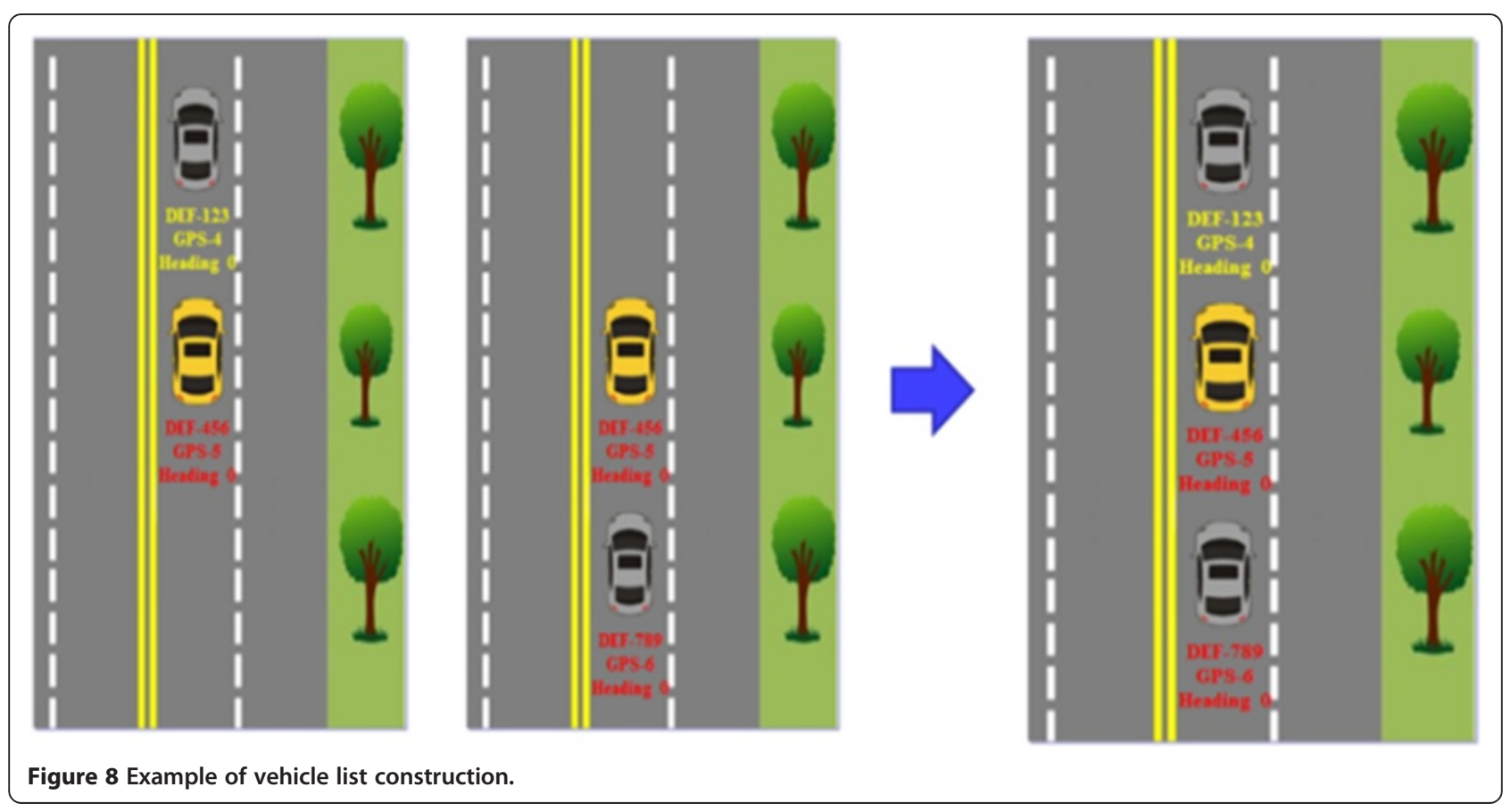




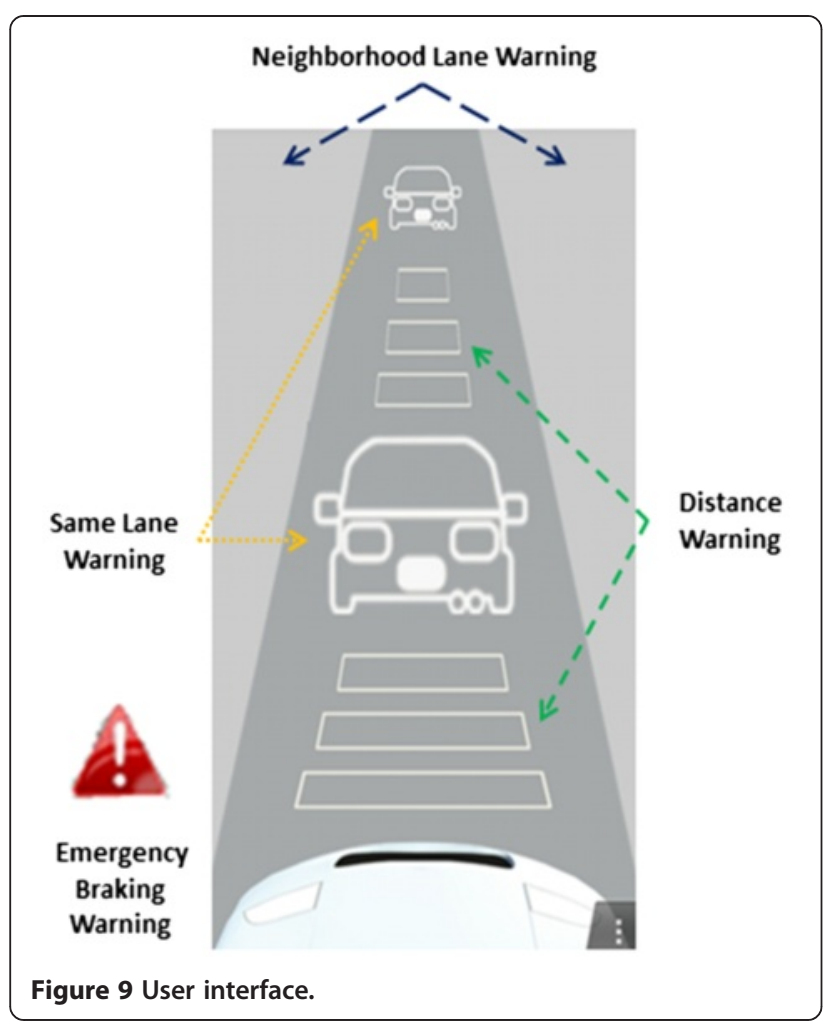

Figure 9 User interface. position accuracy. The DSRC communication is used to alert the driver immediately when a vehicle on the list emergency brakes or a potential emergency event that may hurt drivers occurs. The proposed system allows drivers more reaction time so as to reduce the car accidents on the road.

The emergency braking warning message will broadcast to the neighbouring cars when the car speed decreases $0.1 \mathrm{~g}$ in $0.1 \mathrm{~ms}$. The first car broadcasts the emergency braking warning message, and the second car also broadcasts the emergency braking warning message very soon after. Hence, the third car will receive multiple emergency braking warning messages. We will show the first car and second car emergency braking warning individually in the user interface (Figure 8), because our system uses the vehicle list to identify the car information.

\subsection{Reducing message overhead method}

The middleware of the reducing message overhead method is shown in Figure 11. The IWCU device can be based on the GPS receiver to obtain the position and based on DSRC communication to exchange information with neighbouring cars. The GPS uses NMEA protocol to obtain the latitude, longitude, heading, speed and other information. The above information will feed into the BSM information. The frequency at which the IWCU device broadcasts the BSM information is $10 \mathrm{~Hz}$. The communication protocol is IEEE 1609/IEEE 802.11p

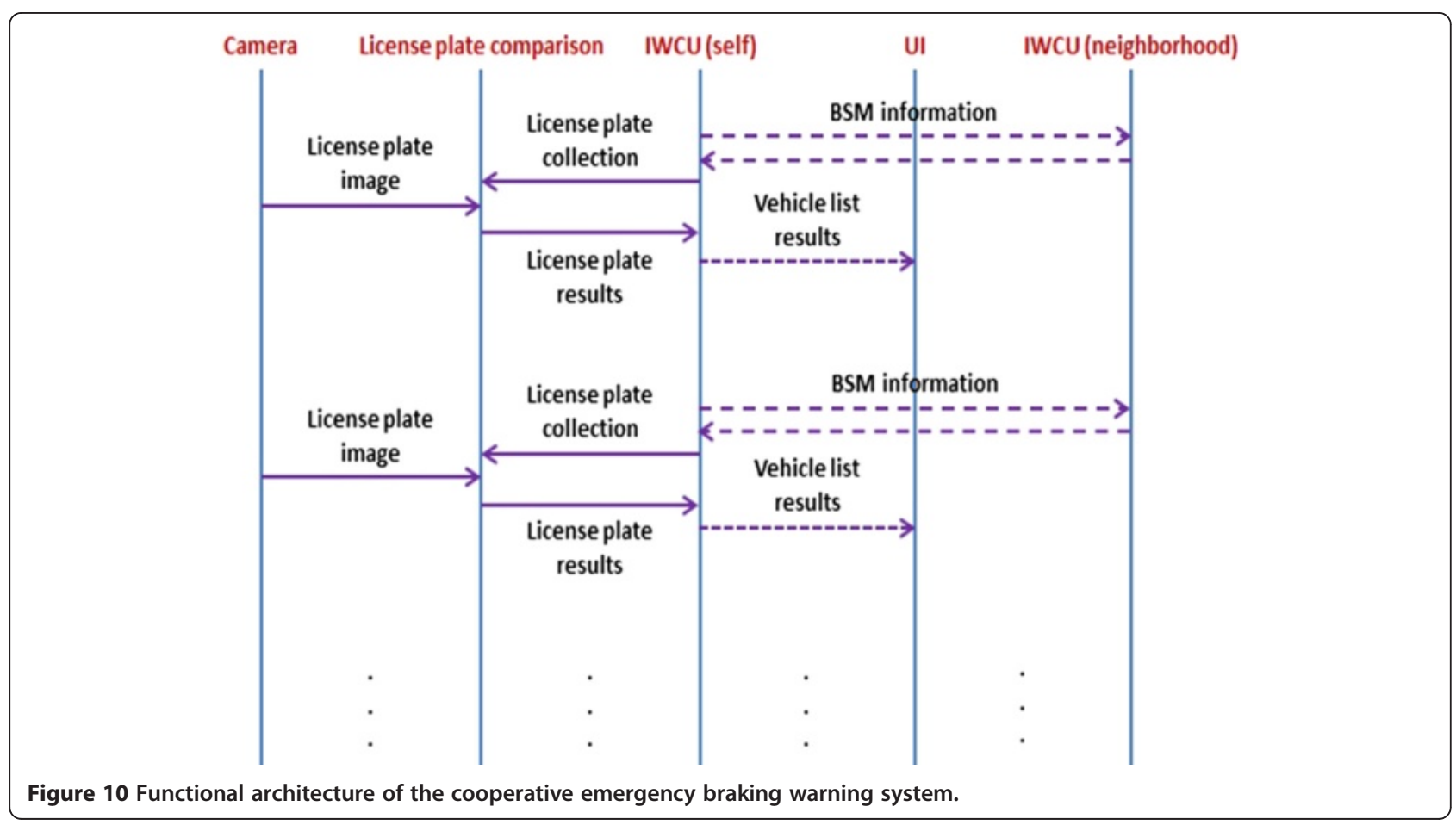




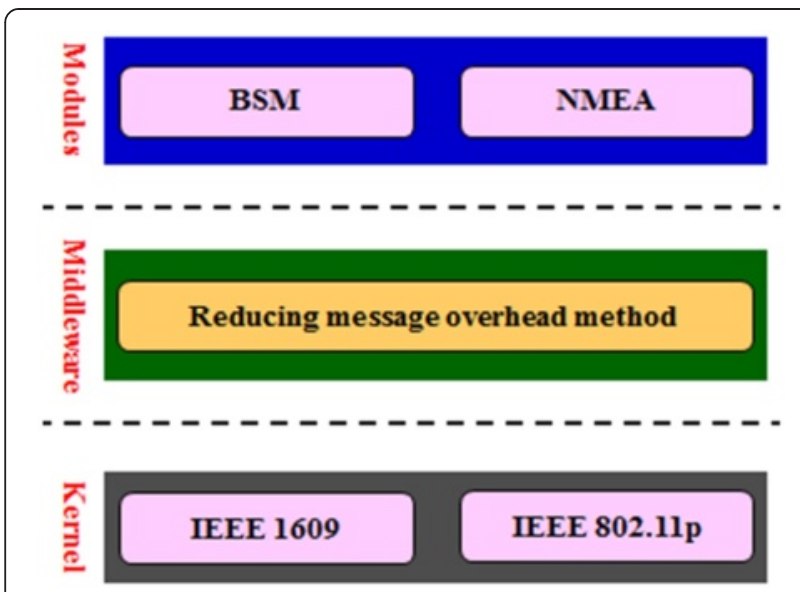

Figure 11 Middleware of the reducing message overhead method.

which provides communication between the self-car and neighbouring cars. The vehicle list (neighbouring cars), built on the BSM information, is shown in Figure 7. For the cooperative emergency braking warning system, the system will warn the driver if the deceleration of neighbouring cars is great and the driving direction is the same (same lane or neighbouring lane). The warning events are based on the speed and heading information of neighbouring cars. This paper reduces the transmitted data by proposing a middleware module called the reducing message overhead method. This method will not affect the original system's operation. This paper focuses on BSM information. The concept of this paper can be applied to other message sets.

The middleware of the reducing message overhead method needs ten fields and includes the BSM information, as shown in Figure 12. The 'lat,' 'long,' 'elev' and 'accuracy' are four bits in pos part. And 'speed', 'heading, 'angle' and 'accelSet' are four bits in motion part. Moreover, 'brakes' is one bit in control part and 'size' is one bit in basic part. This middleware will fill 2 bytes (additional) into the BSM information. The reducing message overhead method is open when the first/second bit

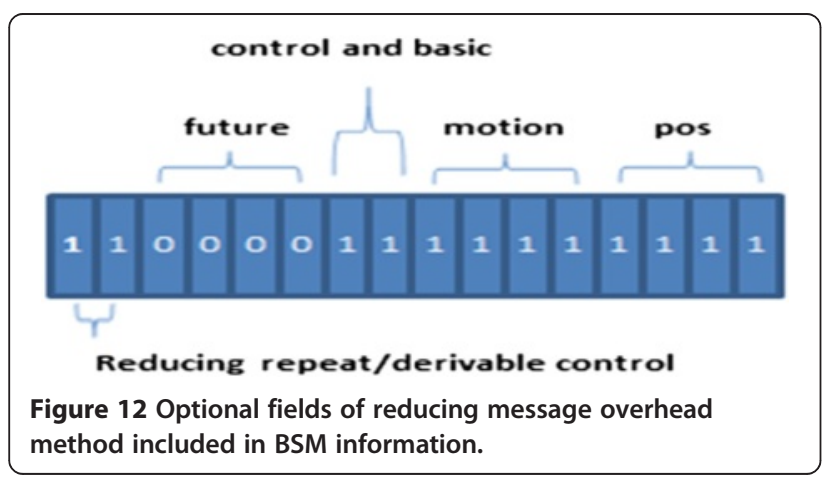

is 1 . The first bit is for the reducing repeat message function, and the second bit is for the reducing derivable message function. Each field of ten fields will not be included in the BSM information when the bit is set to 1 individually. In our proposed method, we need not to obtain the traffic light information. The message should be reduced when the car is waiting at traffic lights, because the information will not change while the car is stationary.

\subsubsection{Reducing repeat message}

The position in the local 3D message uses 16 bytes, which include lat (latitude), long (longitude), elev (elevation) and accuracy (positional accuracy) in the BSM information. These bytes of the message should be reduced when the car is waiting at traffic lights, because the information will not change while the car is stationary. For example, 12,960 bytes of data can be reduced when the red light lasts for $90 \mathrm{~s}$. The motion message in the BSM information uses 12 bytes, which include speed (transmission and speed), heading (heading), angle (steering wheel angle) and accelSet (acceleration set 4 ways). These bytes of the message can also be reduced when the car is waiting at a traffic light, because this message also does not change while stationary. Again, 9,720 bytes of data can be reduced during a 90-s red light. Furthermore, the vehicle size in the BSM information uses 3 bytes. The reducing message overhead method also can reduce the BSM information during unchanged conditions. In the same example, 2,430 bytes of data can be reduced when a red light lasts for $90 \mathrm{~s}$. In the cooperative emergency braking warning system, the control message in the BSM information uses 2 bytes, which are the brakes (brake system status). The warning message does not always happen, so there are 1,620 bytes of data that can be reduced at a 90 -s red light.

\subsubsection{Reducing derivable message}

The local 3D position and motion messages are not independent in the BSM information. Much research work uses the dead reckoning technology to modify the position accuracy, based on the position, speed and heading information to calculate the next position. The reducing derivable message of the reducing message overhead method reduces the motion information in the BSM information. The position of the local 3D message can be used to calculate the motion message in the BSM information which relies on the GPS position between the two points. For example, the local 3D position message in the BSM information will be changed in law when the car moves at a constant speed. The motion message can be derived from the local 3D position message. Hence, the motion message in the BSM information can be reduced for broadcasting information. 


\section{Performance evaluations}

\subsection{Setup and environments}

This paper implemented the cooperative emergency braking warning system and reducing message overhead method in the IWCU devices which were set up as shown in Figure 6. Each car is equipped with the IWCU devices which will broadcast the BSM information frequently at $10 \mathrm{~Hz}$. Hence, the IWCU device will receive neighbouring IWCUs' BSM information by DSRC communication and transmit the information to the smartphone device by Wi-Fi communication. The smartphone device then uses this information to draw out the positions of relevant cars on the screen.

\subsection{Functional results of cooperative emergency braking warning system}

The first function in the cooperative emergency braking warning system is a communication-based forward collision warning, as shown in Figure 13. The speed of three cars is greater than $50 \mathrm{~km} / \mathrm{h}$. The red strip indicates the degree of danger. More red strips means less distance between the self-car and the car in front. The second function in the cooperative emergency braking warning system is an emergency braking warning system in the same lane, as shown in Figure 14. The first car reduces its speed from $50 \mathrm{~km} / \mathrm{h}$ to $0 \mathrm{~km} / \mathrm{h}$. Hence, the third car will receive a communication warning before seeing the second car's brake lights. The third function in the cooperative emergency braking warning system is an emergency braking warning system in a neighbouring lane, as shown in Figure 15. The neighbouring car (first car) reduces its speed from $50 \mathrm{~km} / \mathrm{h}$ to $0 \mathrm{~km} / \mathrm{h}$. Hence, the third car will receive a communication warning shown

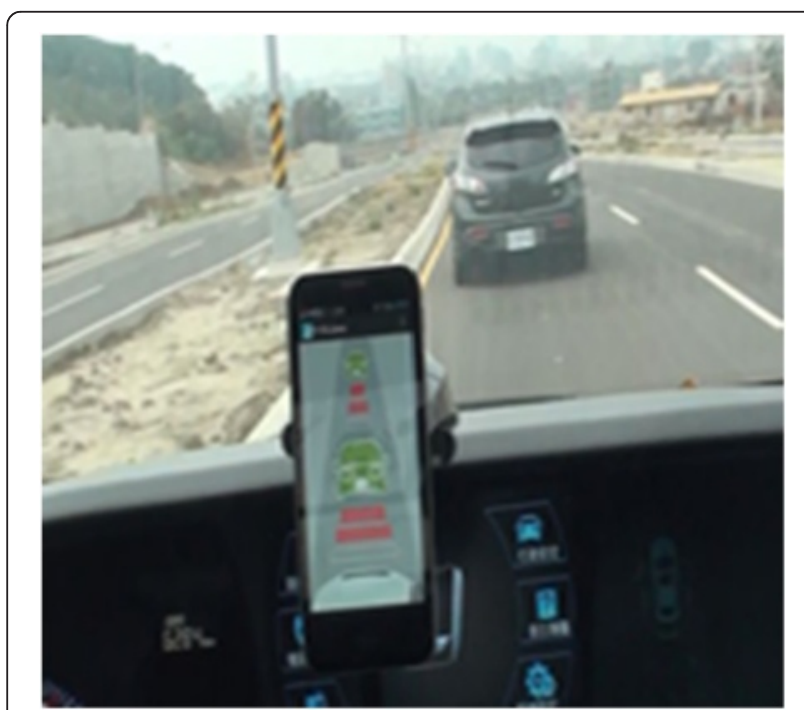

Figure 13 Communication-based forward collision warning.

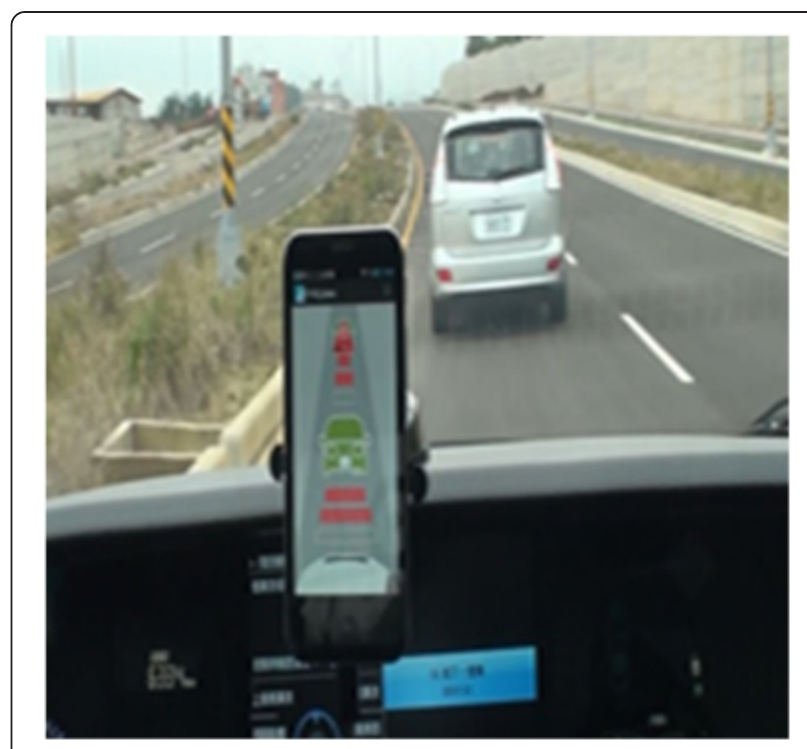

Figure 14 Emergency braking warning system in the same lane.

on the right of the screen before seeing the neighbouring car's brake lights.

\subsection{Performance results of reducing message overhead method}

For the reducing message overhead method, this paper selected two road conditions and counted the total bytes of BSM information. The 'original' column in the chart is without the reducing message overhead method, the 'proposed' column is the reducing message overhead method

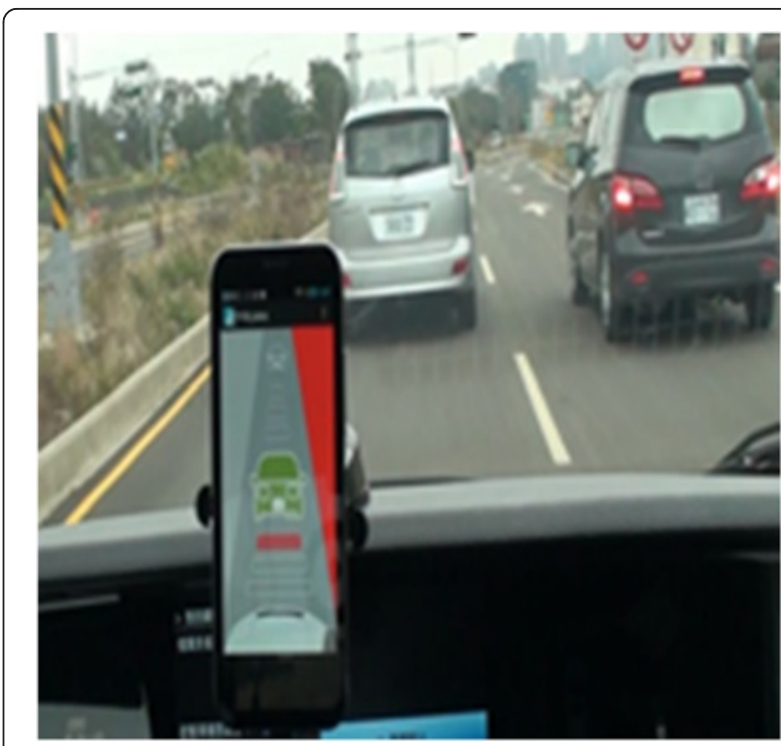

Figure 15 Emergency braking warning system in the neighbouring lane. 


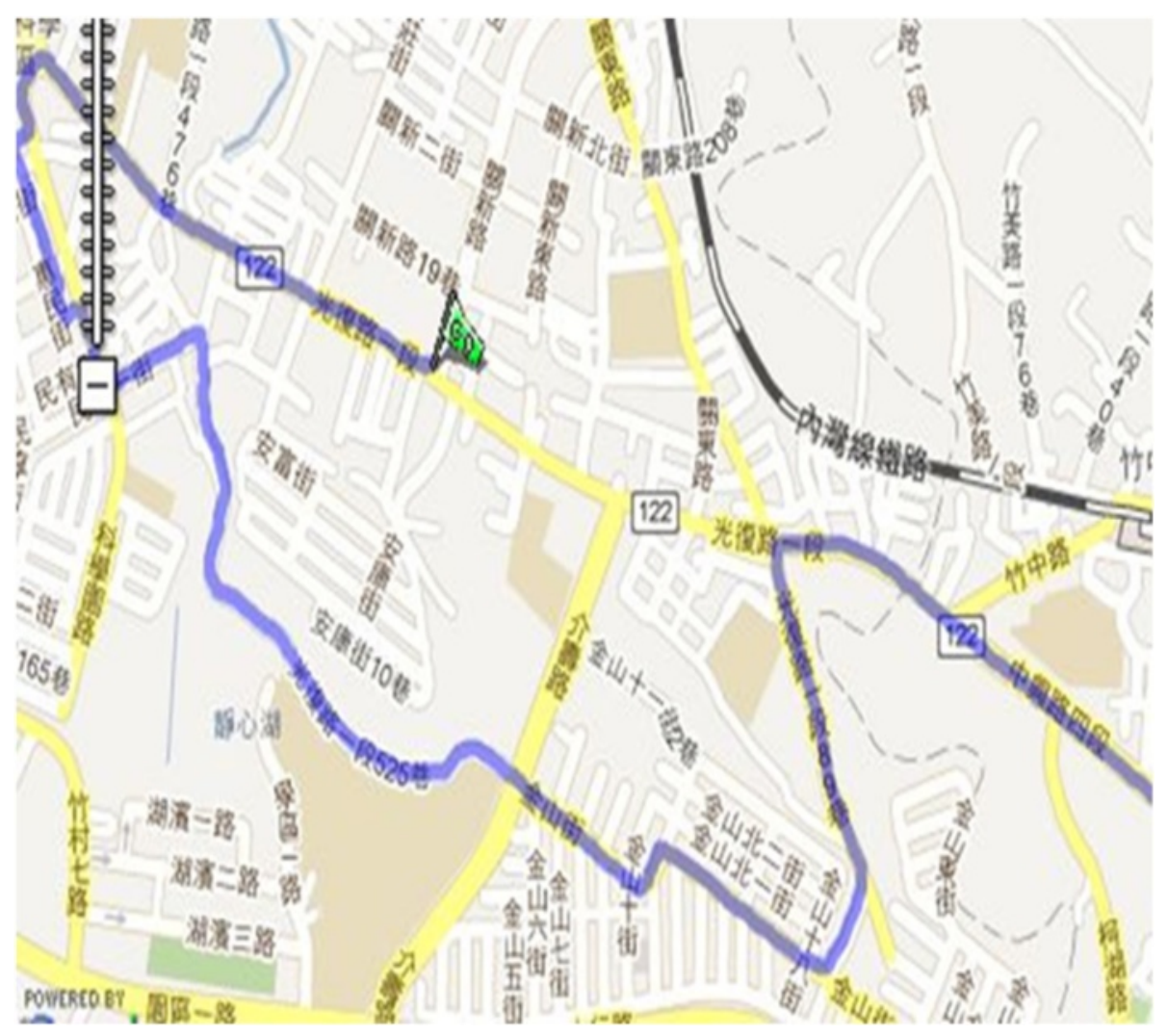

Figure 16 Scenario with traffic light condition.

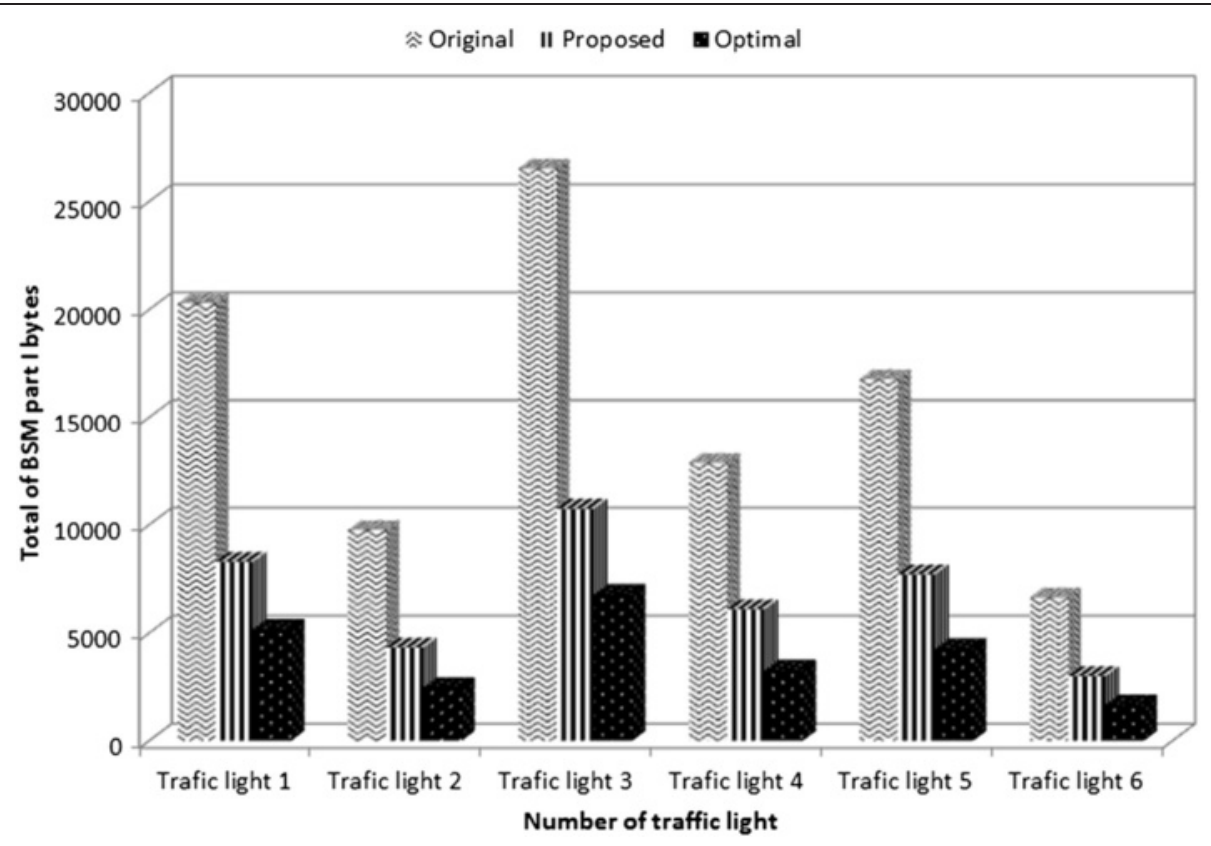

Figure 17 Performance results with waiting at traffic light condition. 


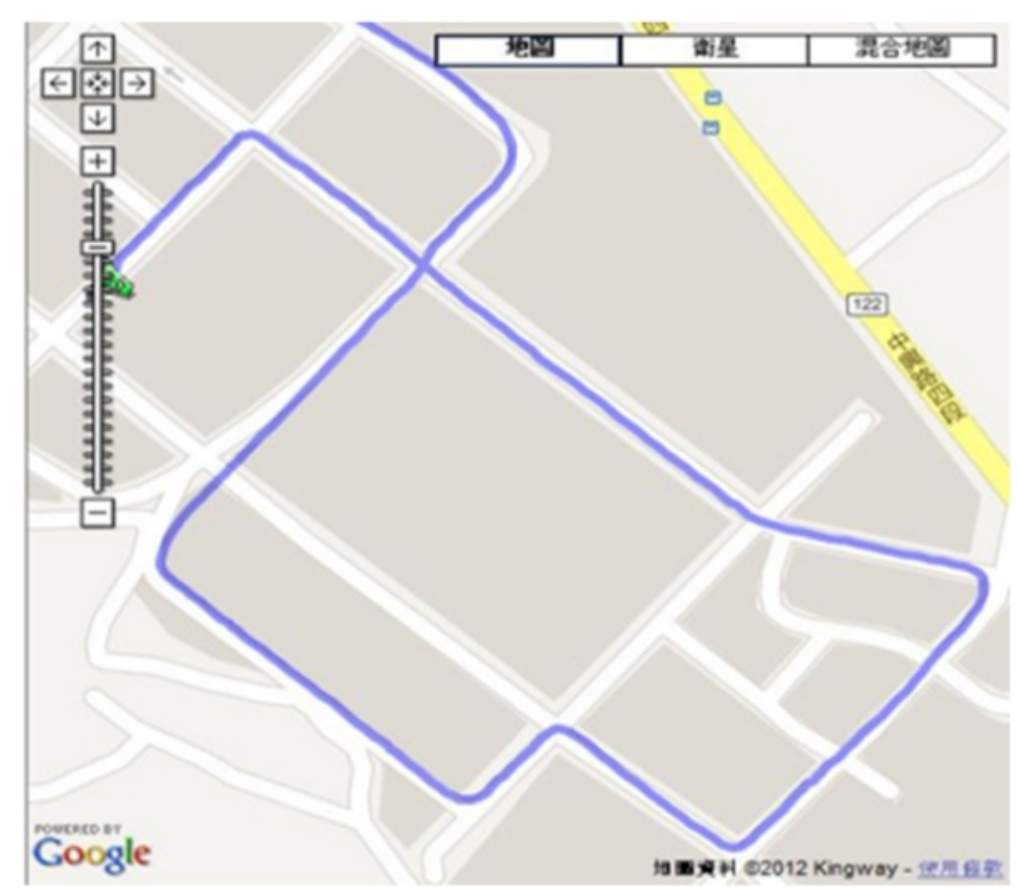

Figure 18 Scenario without traffic light condition.

function opening and the 'optimal' is the reducing message overhead method function working in the best case. The first with a traffic light condition is shown in Figure 16 and provides the performance results of the reducing repeat message function shown in Figure 17. There are two cars moving in tandem in this scenario. The reduced repeat message function will work when the cars are waiting at the traffic light. Hence, the performance results of five conditions when the cars are waiting for the traffic light are shown in Figure 16: traffic light 1 waiting $52 \mathrm{~s}$, traffic light 2 waiting $25 \mathrm{~s}$, traffic light 3 waiting $68 \mathrm{~s}$, traffic light 4 waiting $33 \mathrm{~s}$, traffic light 5 waiting $43 \mathrm{~s}$ and traffic light 6 waiting $17 \mathrm{~s}$ in our experimental scenarios. The total bytes of BSM information are different for each individual car

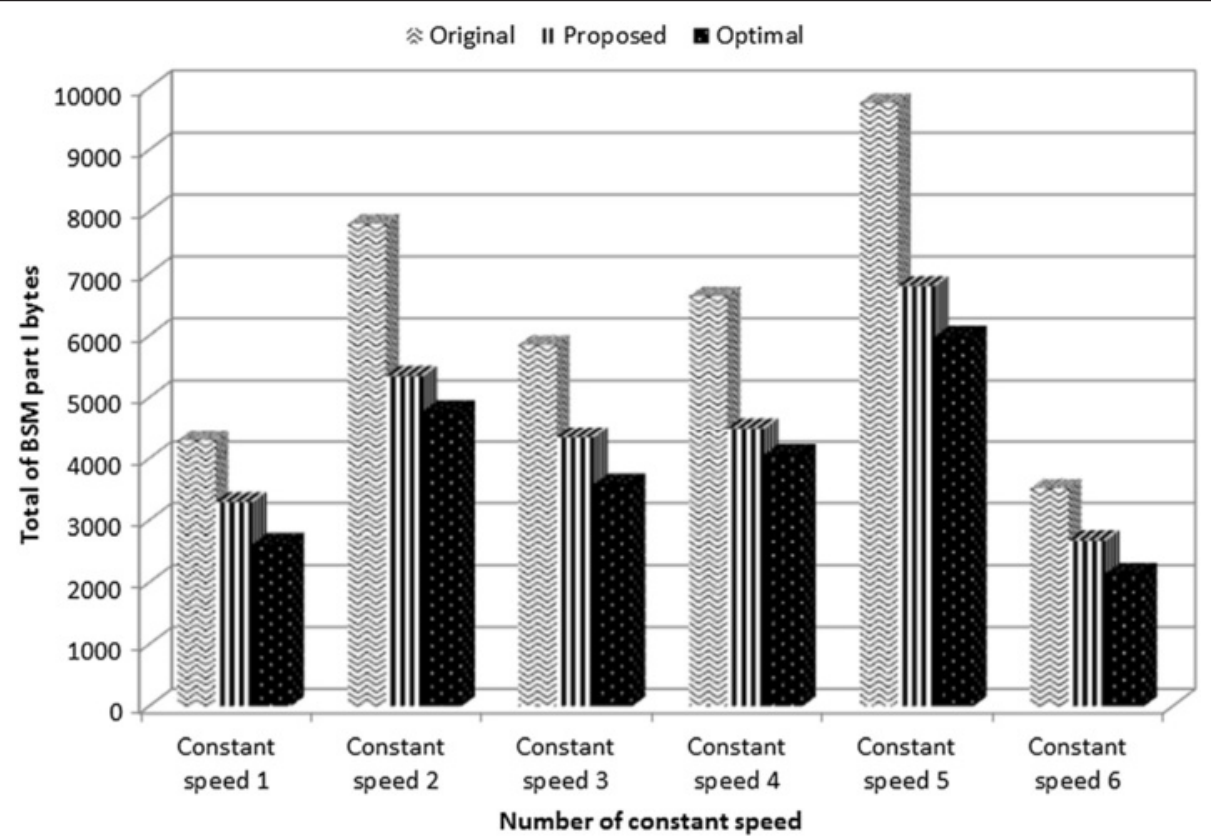

Figure 19 Performance results without waiting at traffic light condition. 
because the waiting time at each traffic light is different. The reducing message overhead method can obtain better performance than the original by reducing repeat messages. However, the performance results of the reducing message overhead method are not perfect (compared to the optimal) because the GPS information will pulsate even when the car is motionless. Hence, the reducing message overhead method will change its on/off high frequency while the car is waiting at the traffic light. However, the performance of the reducing message overhead method will be better when the accuracy of GPS information is enhanced by differential GPS or the wide-area augmentation system.

The second without traffic light condition is shown in Figure 18 and provides the performance results of the reducing derivable message function shown in Figure 19. There are two cars moving in tandem again. However, this scenario keeps the car speed constant during the tests. Constant speed 1 is $30 \mathrm{~km} / \mathrm{h}$ for $11 \mathrm{~s}$, constant speed 2 is $10 \mathrm{~km} / \mathrm{h}$ for $20 \mathrm{~s}$, constant speed 3 is $20 \mathrm{~km} / \mathrm{h}$ for $15 \mathrm{~s}$, constant speed 4 is $20 \mathrm{~km} / \mathrm{h}$ for $17 \mathrm{~s}$, constant speed 5 is $10 \mathrm{~km} / \mathrm{h}$ for $25 \mathrm{~s}$ and constant speed 6 is $30 \mathrm{~km} / \mathrm{h}$ for $9 \mathrm{~s}$ in our experimental scenarios. The reducing derivable message function will work when the cars are travelling at a constant speed. Hence, the performance results of the six conditions under which cars travel at a constant speed are shown in Figure 18. The total bytes of BSM information are different for each individual car because the duration of each constant speed is different. The reducing message overhead method can obtain better performance than the original by reducing the derivable information. However, the performance results of the reducing message overhead method are also not perfect (compared to the optimal) because again the GPS information will pulsate even when the car is travelling at a constant speed. Hence, the reducing message overhead method will change its on/off high frequency when the car travels at a constant speed. Again, the performance of the reducing message overhead method will be better when the accuracy of GPS information is enhanced by other technology. The car is driven in the campus and on city roads in our performance evaluation, hence, the speed and second are restricted by real road conditions. We believe the performance evaluation of our proposed method has similar result when the speed and second are greater.

\section{Conclusion}

Safety applications in vehicular networks have been very important research works in recent years. This paper proposes an integrated camera sensor and DSRC communication to construct the state of neighbouring cars, which is called the cooperative emergency braking warning system. The proposed system is based on the relative position instead of the GPS position in order to solve the positioning accuracy problem. Moreover, the system includes a method for reducing the broadcast message to solve the time-critical problem in vehicular networks. The results of performance evaluations show that the proposed system can provide communication-based forward collision warning, emergency braking warning in the same lane and emergency braking warning in neighbouring lanes. Moreover, the system can reduce the message transmission in order to enhance the effectiveness of transmission information in vehicular networks. The proposed system has been implemented to provide a safer driving environment for drivers.

\section{Competing interests}

The authors declare that they have no competing interests.

\section{Acknowledgement}

This work is supported by the Ministry of Science and Technology of Republic of China (R.O.C) with the project numbered as MOST103-2218-E035-013 and NSC-102-2221-E-035-031-MY3. This research was supported by Basic Science Research Program through the National Research Foundation of Korea(NRF) funded by the Ministry of Education (2013R1A1A2061978).

\section{Author details}

${ }^{1}$ Department of Information Engineering and Computer Science, Feng Chia University, Taichung, Taiwan. ${ }^{2}$ Connected Vehicle Department, Telematics and Vehicular Control System Division, Information and Communications Research Laboratories, Industrial Technology Research Institute, Hsinchu, Taiwan. ${ }^{3}$ Department of Computer Science and Engineering, La Trobe University, Melbourne, Australia. ${ }^{4}$ Department of Multimedia, Sungkyul University, Anyang, Korea.

Received: 22 August 2014 Accepted: 19 January 2015 Published online: 19 February 2015

\section{References}

1. T Hsieh, Y Chao, P Tsai, M Tsai, Real-time driving safety of dynamic full screen bird view technology and applications. ICL J. 153(6), 43-50 (2013)

2. H Song, $\mathrm{H}$ Lee, An Overview of Analytic Models of the IEEE 802.11p MAC Protocol for Vehicle-to-Vehicle Safety Communications and Their Technical Issues (IEEE International Conference on ICT Convergence, Korea, 2013), pp. 612-613

3. M Tsai, Cooperative emergency electronic brake warning system with radar detection. ICL J. 151(8), 59-64 (2013)

4. P Alexander, D Haley, A Grant, Cooperative intelligent transport systems: 5.9-GHz field trials. Proc. IEEE. 99(7), 1213-1235 (2011)

5. M Tsai, N Chilamkurti, P Ho, Y Lu, Implementation of dedicated short range communications combined with radar detection for forward collision warning system. Int. J. Wireless Netw. Broadband Technol. 2(1), 49-63 (2012)

6. U.S. DOT announces decision to move forward with vehicle-to-vehicle communication technology for light vehicles, http:/www.dot.gov/briefingroom.

7. P Szczurek, B Xu, O Wolfson, J Lin, Estimating relevance for the emergency electronic brake light application. IEEE Trans. Intell. Transportation Syst. 13(4), 1638-1656 (2012)

8. M Li, T Wu, W Lin, K Lan, C Chou, C Hsu, On the Feasibility of Using 802.11p for Communication of Electronic Toll Collection Systems (IEEE International Conference on Network-Based Information Systems, Albanic, 2011), pp. 68-75

9. Y Park, H Kim, Collision control of periodic safety messages with strict messaging frequency requirements. IEEE Trans. Vehicul. Technol. 62(2), 843-852 (2013)

10. The CAMP Vehicle Safety Communications Consortium, Vehicle Safety Communications Project Task 3 Final Report: identify intelligent vehicle safety applications enabled by (DSRC U.S. Department of Transportation, Washington, DC, USA, 2005)

11. N Alam, AT Balaei, AG Dempster, A DSRC Doppler-based cooperative positioning enhancement for vehicular networks with GPS availability. IEEE Trans. Vehicul. Technol. 60(9), 4462-4470 (2011) 
12. B Kim, I Kang, H Kim, Resolving the unfairness of distributed rate control in the IEEE WAVE safety messaging. IEEE Trans Vehicular Technol. doi:10.1109/TVT.2013.2290373.

13. Y Park, H Kim, Application-level frequency control of periodic safety message in the IEEE WAVE. IEEE Trans. Vehicul. Technol. 61(4), 1854-1862 (2012)

14. M Tsai, C-H Chang, A Reducing Broadcast Message Method in Vehicular Networks. (Kyushu, Japan, IET International Conference on Communications and Robotics, 2014-08), pp. 1-4

15. IEEE, IEEE Standard for Wireless Access in Vehicular Environments (WAVE) Networking Service (IEEE Standard 1609.3, Piscataway, NJ, USA, 2010)

16. Wikipedia, Differential GPS. http://en.wikipedia.org/wiki/Differential_GPS

17. Wikipedia, Wide area augmentation system. http://en.wikipedia.org/wiki/ Wide_Area_Augmentation_System.

18. C Campolo, A Vinel, A Molinaro, Y Koucheryavy, Modeling broadcasting in IEEE 802.11 p/WAVE vehicular networks. IEEE Commun. Lett. 15(2), 199-201 (2011)

19. M Torrent-Moreno, P Santi, H Hartenstein, Fair Sharing of Bandwidth in VANETS (ACM International Workshop on Vehicular Ad Hoc Networks, Germany, 2005), pp. 49-58

20. J Mittag, F Schmidt-Eisenlohr, M Killat, J Harri, H Hartenstein, Analysis and Design of Effective and Low-Overhead Transmission Power Control for VANETS (ACM International Workshop on Vehicular Internetworking, USA, 2008), pp. $39-48$

21. L Wei, X Xiao, Y Chen, M Xu, H Fan, Power-control-based Broadcast Scheme for Emergency Messages in VANETS (IEEE International Symposium on Communications and Information Technologies, China, 2011), pp. 274-279

22. M Tsai, J Lin, Improving License Plate Recognition Performance Using Inter-Vehicle Communications (Symposium on Digital Life Technologies, Taiwan, 2014), pp. 1-4

\section{Submit your manuscript to a SpringerOpen ${ }^{\circ}$ journal and benefit from:}

- Convenient online submission

- Rigorous peer review

- Immediate publication on acceptance

- Open access: articles freely available online

- High visibility within the field

- Retaining the copyright to your article

Submit your next manuscript at $\boldsymbol{\wedge}$ springeropen.com 